\title{
Validation of Skinfold Measurement Method to Dexa for the Assessment of Body Composition
}

\author{
Umid Karli, Yilmaz Ucan, Kerim Sozbir **, Kutlu Aydin, Hakan Yarar \\ Coaching Education, School of Physical Education and Sport, University of Abant Izzet Baysal, \\ Golkoy Kampusu, Bolu/TURKEY. \\ umidkarli@gmail.com,mr_ucan@hotmail.com,sozbir_k@ibu.edu.tr,kutlu_aydin@yahoo.com, \\ hakanyarar96@mynet.com
}

\begin{abstract}
:
Introduction: Skinfold (SKF) measurement provides an affordable and practical assessment of body fat percentage (BF\%) and fat free mass (FFM) in field-based studies. However, SKF measurement is relatively safe, quick, cheap and non-invasive method (21) for the assessment of body composition, use of inappropriate equations in SKF measurement method can lead systematic or non-systematic prediction errors (25).
\end{abstract}

Purpose: The purpose of this study was to compare the BF\% and FFM value obtained by the SKF measurement method and dual-energy $X$-ray absorptiometry (DEXA) considered as a reference method.

Methods: Fifty two male (mean $\pm S D$, age: $25.23 \pm 1.60 y$; stature: $175.05 \pm 5.86 \mathrm{~cm}$; body weight: $72.53 \pm$ $9.75 \mathrm{~kg}$ ) and forty six female (mean $\pm S D$, age: $24.54 \pm 2.14 y$ r; stature: $165.88 \pm 6.13 \mathrm{~cm}$; body weight: $57.60 \pm$ $7.17 \mathrm{~kg}$ ) Caucasian sedentary were participated in this study. Each participant's BF\% and FFM was assessed both by SKF measurement method and reference measurement DEXA. Widely used equations of Durnin and Womersley (20) and Siri (27) were used to calculate BF\% from SKF measurements. All measurements were performed at least three hours after lunch between 15:00 and 17:00 o'clock.

Results: Results of paired t test revealed that there was significant difference between SKF and DEXA measurement methods regarding to BF\% $(t=-6.785 ; p=0.001)$ and FFM $(t=6.568 ; p=0.001)$ values of males. Likewise, significant difference was indicated between SKF and DEXA measurement methods with respect to $B F \%(t=-6.126 ; p=0.001)$ and FFM $(t=6.363 ; p=0.001)$ values also in female participants. On the other hand, the BF\% and FFM values obtained by using SKF method were highly correlated with those obtained by DEXA in males (BF\%: $r=0.894, p=0.001 ; S E E=2.715 \%$ and FFM: $r=0.923, p=0.001 ; S E E=2.111 \mathrm{~kg})$ and females (BF\%: $r=0.883, p=0.001 ;$ SEE = 3.197\% and FFM: $r=0.838, p=0.001 ; S E E=2.044 \mathrm{~kg}$ ), respectively.

Conclusion: Accordingly, subgroups of Caucasian population-specific equations should be developed for accurate estimation of BF\% for Turkish population.

Keywords: Validity, Body Density, Body Fat Percentage and Fat Free Mass.

\section{INTRODUCTION}

Body composition is the health-related component of physical fitness (1) and is widely used by physicians, athletics trainers and health professionals $(2,3)$. Measurements of body composition provide important information of the various components of the human body such as fat mass, fat-free mass (FFM) and body fat percentage (BF\%) (4). Different techniques have been used to the measurement of body composition are currently available which differ in various criteria, including sensitivity, cost, precision, invasiveness and technical complexity (5). Hydrostatic weighing, air displacement plethysmography and dual-energy X-ray absorptiometry (DEXA) are considered reference methods to assess body composition $(6,7,8)$.

Previously, hydrostatic weighing was considered as the gold standard for the assessment of body composition but this method had many technical complexity $(4,9)$, inconvenient and difficult to use (10) and also demands a long time (11). Thus, other laboratory techniques have been developed and improved to BF\% estimate (12). DEXA seems to be an alternative to the problems presented by hydro densitometry and allows for a rapid evaluation and with reliable data when comparing to hydrostatic weighting (7). Currently, DEXA has been considered as the gold standard for the measurement of 
body composition (9). DEXA has been shown to be an effective measure of body composition and has been considered a valid and reliable reference measure in many populations $(7,13,14,15)$. On the other hand, reference techniques are routinely expensive, non-portable requires sophisticated laboratory equipment and is quite time-consuming and also often impractical for field-based studies $(8,16,17,18)$.

Skinfold (SKF) thickness measurement provides an affordable and practical assessment of BF\% and FFM in large populations in field-based studies $(8,19)$. This technique was a recommended method for a long time for determining BF\% (20) and also FFM. SKF measurement is relatively safe and provides a rapid result for studying different populations, without the need for specialized laboratory equipment, exposure to radiation or costly equipment for the assessment of body composition $(21,22$, 23). However, equations used to predict total body fat from subcutaneous SKF measurement are based on adult cadaver studies of small sample sizes (24) and may not be relevant or valid in different populations. Furthermore, SKF measurements only provide an estimate of subcutaneous fat (10) and can not be used to reliably measure fat stored internally such as visceral fat (15). Moreover, the use of inappropriate equations in SKF measurement method can lead systematic or non-systematic prediction errors (25). Therefore, the purpose of this study was to compare the BF\% and FFM value obtained by the SKF measurement method and DEXA considered as reference method.

\section{Materials AND Methods}

Fifty two male (mean \pm SD, age: $25.23 \pm 1.60 \mathrm{yr}$; stature: $175.05 \pm 5.86 \mathrm{~cm}$; body weight: $72.53 \pm$ $9.75 \mathrm{~kg}$ ) and forty six female (mean $\pm \mathrm{SD}$, age: $24.54 \pm 2.14 \mathrm{yr}$; stature: $165.88 \pm 6.13 \mathrm{~cm}$; body weight: $57.60 \pm 7.17 \mathrm{~kg}$ ) Caucasian sedentary volunteered to participate in this study. The participants were fully informed about the protocols and procedures of the study before participating in the study.

\subsection{Study Design}

All measurements were performed at least three hours (h) after lunch at the same time of the day between 15:00 and 17:00 o'clock. At first, anthropometric and SKF measurements were completed respectively. Then, DEXA measurements were applied in order to collect reference values. Tests were conducted at a constant environmental temperature and humidity $\left(20-23^{\circ} \mathrm{C}\right.$ and $50-60 \%$, respectively). The participants were instructed to avoid exercise for a minimum of $24 \mathrm{~h}$ before testing.

\subsection{Anthropometric Measurements}

All the measurements were made following the guidelines outlined by the International Society for the Advancement of Kinanthropometry (ISAK) (26) by the same experienced investigator.

Body mass was measured with a Seca scale (Seca 700, Medical Scales and Measuring Systems, Hamburg-GERMANY; precision of $0.1 \mathrm{~kg}$ ) and with the participant wearing underwear only and barefoot. Height was measured with a stadiometer incorporated into the scale (Seca 700, Medical Scales and Measuring Systems, Hamburg-GERMANY; precision of $0.1 \mathrm{~cm})$. The participant was instructed to take and hold a full breath. Stretched height with the head at the Frankfort plane was recorded.

\subsection{Skinfold Measurement}

SKF Measurements were performed following the protocol of the ISAK by the same trained and experienced investigator for each participant (26). SKF thickness was measured on the right side of the body to the nearest $\pm 0.1 \mathrm{~mm}$ with a SKF caliper (Holtain Ltd, Crymych, UK) from following sites.

a) Subscapular, about $20 \mathrm{~mm}$ below the inferior angle of the scapula and $45^{\circ}$ to lateral side of the body (Figure 1a).

b) Triceps, a vertical fold halfway between the acromion process and the superior head of the radius, in the posterior aspect of the arm (Figure 1b).

c) Biceps, at the same level as the triceps skinfold and directly above the centre of the cubital fossa (Figure 1c).

d) Iliac Crest, immediately above the marked illiocrestale (the point on the most lateral aspect of the iliac tubercle, which is on the iliac crest) (Figure 1d). 
SKF measurements were taken twice or three times (if the difference between the first two measures was greater than $5 \%$ ) with the mean or median values for each site in a rotational order, respectively. The equation of Durnin and Womersley (20) was used to calculate the density of body from the SKF thickness variables. The density value was then converted to BF\% using the Siri equation (27). FFM was calculated by subtracting fat mass from body mass.

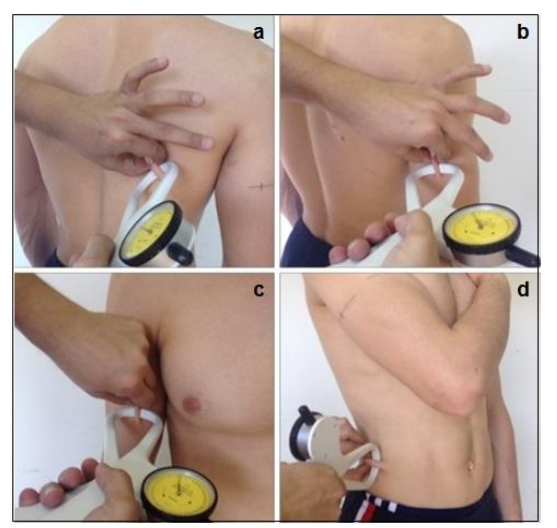

Figure1. SKF Thickness Measurement Sites. (a) Subscapular, (b) Triceps, (c) Biceps, (d) Iliac Crest.

\subsection{DEXA Measurements}

For the DEXA method, body composition was assessed by a total body scanner, using the Lunar DPX-IQ model (Lunar Radiation Corporation, Madison, WI, USA). All DEXA scans were performed by the same technician, who was trained in the operation of the scanner, the positioning of participants, and the analysis of results. Whole body scanning took about 20 minutes related to the participant's stature. The average skin entrance dose was $0.015 \mathrm{mrem}$. The results are presented as $\mathrm{BF} \%$ and FFM was calculated by subtracting fat mass from body mass. All measurements were carried out at the University of Abant Izzet Baysal.

\subsection{Statistical Analysis}

After the calculation of mean and standard deviations (SD) for all variables, paired samples test was used to compare the values (BF\% and FFM) obtained from DEXA and SKF. Simple linear regressions were performed to calculate the relationship between BF\% and FFM estimated by the reference measurement DEXA and SKF. Significance level of alpha was accepted as 0.05 and all analyses were performed on statistical package for the social sciences (SPSS version 16.0, SPSS Inc., Chicago, IL, USA).

\section{RESUlTS}

Results of paired $t$ test revealed that there was significant difference between SKF and DEXA measurement methods regarding to $\mathrm{BF} \%(\mathrm{t}=-6.785 ; \mathrm{p}=0.001)$ and $\mathrm{FFM}(\mathrm{t}=6.568 ; \mathrm{p}=0.001)$ values of males. Likewise, significant difference was indicated between SKF and DEXA measurement methods with respect to $\mathrm{BF} \%(\mathrm{t}=-6.126 ; \mathrm{p}=0.001)$ and $\mathrm{FFM}(\mathrm{t}=6.363 ; \mathrm{p}=0.001)$ values also in female participants.

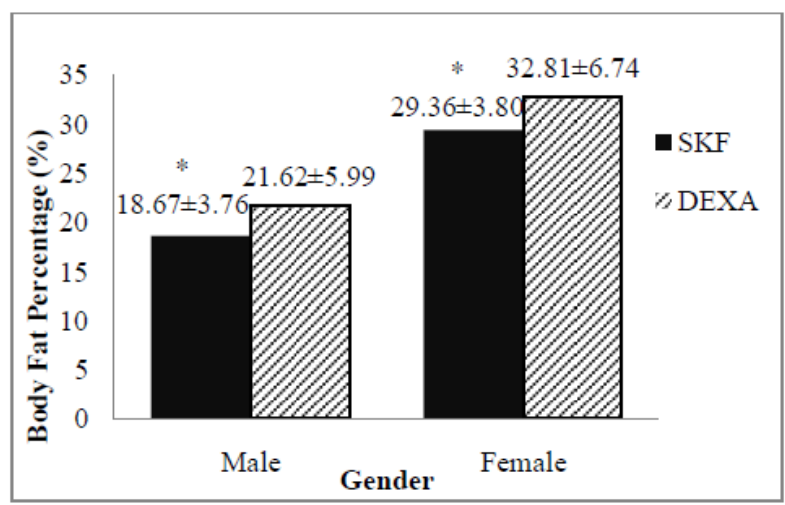

Figure2. BF\% Values of Male and Female Participants Determined Via SKF and DEXA (* Significant Different than DEXA) 


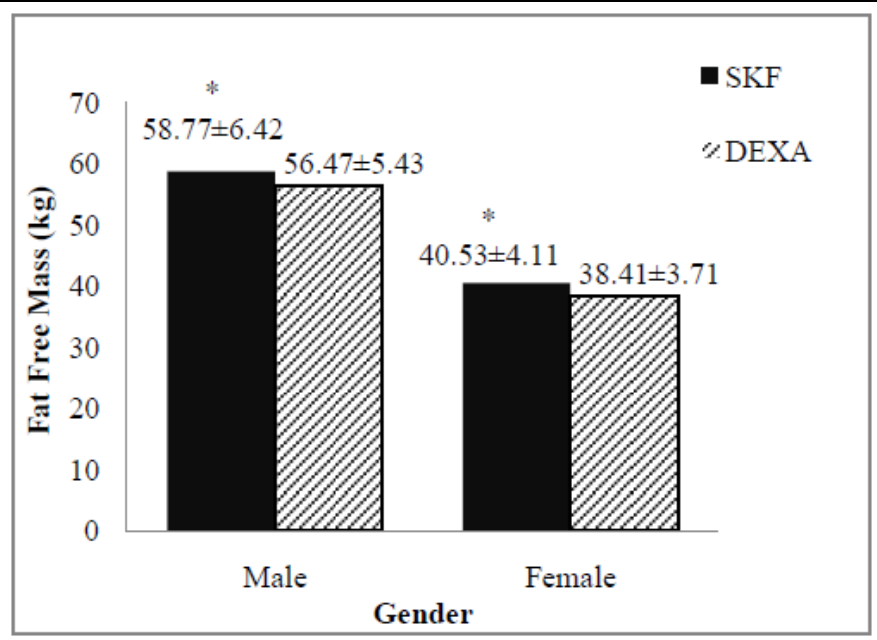

Figure3. FFM Values of Male and Female Participants Determined Via SKF and DEXA (* Significant Different than DEXA)

The BF\% and FFM values obtained using SKF were related with those obtained from DEXA in males (BF\%: $r=0.894, p=0.001$ and FFM: $r=0.923, p=0.001)$ and females $(r=0.883, p=0.001$ and FFM: $r=$ $0.838, \mathrm{p}=0.001)($ Table 1 and 2).

Table1. Validation of SKF to DEXA Via BF\% in Males and Females

\begin{tabular}{|l|c|}
\hline Validation results for males & \\
\hline Constant error DEXA-SKF $(\%)$ & $2.945 \pm 3.130$ \\
\hline r(DEXA vs. SKF) & 0.894 \\
\hline SEE for SKF $(\%)$ & 2.715 \\
\hline Validation results for females & \\
\hline Constant error DEXA-SKF $(\%)$ & $3.450 \pm 3.820$ \\
\hline r(DEXA vs. SKF) & 0.883 \\
\hline SEE for SKF $(\%)$ & 3.197 \\
\hline
\end{tabular}

Table2. Validation of SKF to DEXA Via FFM in Males and Females

\begin{tabular}{|l|c|}
\hline Validation results for males & \\
\hline Constant error DEXA-SKF $(\mathrm{kg})$ & $2.299 \pm 2.524$ \\
\hline r(DEXA vs. SKF) & 0.923 \\
\hline SEE for SKF $(\mathrm{kg})$ & 2.111 \\
\hline Validation results for females & $2.118 \pm 2.258$ \\
\hline Constant error DEXA-SKF (kg) & 0.838 \\
\hline r(DEXA vs. SKF) & 2.044 \\
\hline SEE for SKF $(\mathrm{kg})$ & \\
\hline
\end{tabular}

However, the correlation between BF\% obtained by SKF measurement and DEXA is high $(\mathrm{r}=0.894$; $r=0.883$ for males and females, respectively), SKF measurement method underestimated BF\% both in males and females compared to DEXA. As BF\% the correlation between FFM obtained by SKF measurement and DEXA is also high $(\mathrm{r}=0.923 ; \mathrm{r}=0.838$ for males and females, respectively). However, opposite to BF\% FFM was overestimated by SKF measurement method both in males and females compared to DEXA.

\section{DiscuSSION AND CONCLUSIONS}

This study was designed for the comparison of the BF\% and FFM value obtained by the SKF measurement method and DEXA considered as reference method. The major finding of this study is the presence of the difference between SKF measurement method and DEXA with respect to BF\% and FFM both in males and females. On the other hand, again regarding to BF\% and FFM, the relation between SKF measurement and DEXA is quite high.

$\mathrm{BF} \%$ values obtained by SKF measurement method was significantly $(\mathrm{p}=0.001)$ lower than the reference measurement DEXA both for male and female sedentary participants (Figure 2). Meanwhile, the correlation between BF\% obtained by SKF measurements and DEXA is high and, SEE for BF\% values of SKF is relatively small (Table 1). According to the American College of 
Sports Medicine (28), obesity is associated with increased risk for the development of cardiovascular disease, hypertension, diabetes, specific types of cancer and other chronic diseases. Therefore, an accurate assessment of one's body composition is important for all individuals, as BF\% is closely related to one's health and well-being (19). There are many equations in the literature that predict body fatness from SKF measurements (29). In the present study, the predicted values obtained from SKF thickness measurements were calculated by the equations of Durnin and Womersley (20) and Siri (27). The most of the body composition prediction equations have been developed for Caucasian populations-specific who were from European descent. However, the participants included in the present study are Caucasian but also are a homogeneous amalgamation of different ethnicity from three continents (European, African and Asian ancestry). Variation of the ethnicity might be the possible explanation of the difference between SKF values and DEXA regarding to BF\%. Hence, ethnicity is one of the major factors that effect the fat distribution of the body, especially the adipose tissue located under the skin which is called as subcutaneous fat influences the thickness of the SKF. The measurement sites of the Durnin and Womersley (20) equation used in this study may not be suitable for accurate estimation of the body composition parameters of the present participants. The possible explanation for the underestimation of the SKF method might be the predictor variables used for calculation of the $\mathrm{BF} \%$ in these equations.

FFM values calculated via BF\% values obtained by SKF measurement method was significantly $(\mathrm{p}=$ 0.001) higher than the reference measurement DEXA both for male and female sedentary participants (Figure 3). Meanwhile, the correlation between FFM obtained from the SKF measurement method and DEXA is high and, SEE for FFM values of SKF is relatively small (Table 2). The variation of the ethnicity might be the possible explanation also for the difference between FFM obtained from SKF method and DEXA. The possible explanation for the overestimation of FFM obtained from SKF method might be the predictor variables used for calculation of the BF\% in these equations.

Whether the correlation between BF\% and FFM obtained by SKF measurement and DEXA is high, SKF measurement method underestimated BF\% and overestimated FFM values both in males and females compared to DEXA. The data as an outcome of this study indicated that SKF method presented no expected accuracy in estimating BF\% and FFM for Turkish population either for males or females in relation with the DEXA reference method. Hence, subgroups of Caucasian populationspecific equations should be developed for accurate estimation of BF\% and FFM for Turkish population.

\section{ACKNOWLEDGEMENT}

The authors grateful to participants who participated in the study. This article presented as a poster presentation at " $6^{\text {th }}$ Annual International Conference: Physical Education, Sport and Health" in Pitesti/ROMANIA. No sources of funding were used to assist in the preparation of this study. The authors declare that they do not have any conflict of interest in accordance with the journal policy and disclose no professional relationships with companies or manufacturers who will benefit from the results of the present study.

\section{REFERENCES}

[1] Warner, E.R., Fornetti, W.C., Jallo, J.J. and Pivarnik, J.M., A Skinfold Model to Predict Fat-Free Mass in Female Athletes. J Athl Training, 39(3), 259-262 (2004).

[2] Steiner, M.C., Barton, R.L., Singh, S.J. and Morgan M.D.L., Bedside methods versus dual energy X-ray absorptiometry for body composition measurement in COPD. Eur Respir J, 19, 626-631(2002).

[3] Clark, R.R., Bartok, C.,Sullivan, J.C. and Schoeller, D.A., Minimum weight prediction methods cross-validated by the four-component model. Med \& Sci in Sports \& Exerc, 36(4), 639-647 (2004).

[4] Lara, J., Johnstone, A.M., Wells, J., Jebb, S. and Siervo, M., Accuracy of aggregate 2-and 3component models of body composition relative to 4-component for the measurement of changes in fat mass during weight loss in overweight and obese subjects. Applied Physiology, Nutrition, and Metabolism, 39(8), 871-879 (2014).

[5] Lee, S.Y. and Gallagher, D., Assessment methods in human body composition. Current opinion in clinical nutrition and metabolic care, 11(5), 566-572 (2008). 
[6] Fields, D.A. and Goran, M.I., Body composition techniques and the four-compartment model in children. Journal of Applied Physiology, 89(2), 613-620 (2000).

[7] Rech, C.R., Petroski, E.L., Silva, R.C.R.D. and Silva, J.C.N.D., Anthropometric indicators of fat mass excess among women. Revista Brasileira de Medicina do Esporte, 12(3), 119-124 (2006).

[8] Espana Romero, V., Ruiz, J.R., Ortega, F.B., Artero, E.G., Guez, G.V.R., Moreno, L.A., Castillo, M.J. and Gutierrez, A., Body fat measurement in elite sport climbers: Comparison of skinfold thickness equations with dual energy X-ray absorptiometry. J Sports Sci, 27(5), 469-477 (2009).

[9] Gupta, N., Balasekaran, G., Govindaswamy, V.V., Hwa, C.Y. and Shun, L.M., Comparison of body composition with bioelectric impedance (BIA) and dual energy X-ray absorptiometry (DEXA) among Singapore Chinese. J Sci Med Sport, 14, 33-35 (2011).

[10] Vasquez, F., Diaz, E., Lera, L., Vasquez, L., Anziani, A. and Burrows, R., Agreement of anthropometric equations with the 4-component model in the prediction of body fat in obese schoolchildren. Nutrition \& Dietetics, 69(2), 145-151 (2012).

[11] Brodowicz, G.R., Mansfield, R.A., Mcclung, M.R. and Althoff, S.A., Measurement of body composition in the elderly: dual energy x-ray absorptiometry, underwater weighing, bioelectrical impedance analysis, and anthropometry. Gerontology, 40(6), 332-339 (1994).

[12] Kohrt, W.M., Preliminary evidence that DEXA provides an accurate assessment of body composition. Journal of applied physiology, 84(1), 372-377 (1998).

[13] Kamimura, M.A., Avesani, C.M., Cendoroglo, M., Canziani, M.E.F., Draibe, S.A. and Cuppari, L., Comparison of skinfold thicknesses and bioelectrical impedance analysis with dual-energy Xray absorptiometry for the assessment of body fat in patients on long-term haemodialysis therapy. Nephrol Dial Transplant, 18, 101-105 (2003).

[14] Lintsi, M., Karma, H. and Kull, I., Comparison of hand-to-hand bioimpedance and anthropometry equations versus dual-energy X-ray absorptiometry for the assessment of body fat percentage in 17-18-year-old conscripts. Clin Physiol Funct Imaging, 24, 85-90 (2004).

[15] Watts, K., Naylor, L.H., Davis, E.A., Jones, T.W., Beeson, B., Bettenay, F., Siafarikas, A., Bell, L., Ackland, T. and Green, D.J., Do skinfolds accurately assess changes in body fat in obese children and adolescents? Medicine and science in sports and exercise, 38(3), 439-444 (2006).

[16] Jones, L.M., Goulding, A. and Gerrard, D., DEXA: a practical and accurate tool to demonstrate total and regional bone loss, lean tissue loss and fat mass gain in paraplegia. Spinal Cord, 36, 637-640 (1998).

[17] Duz, S., Kocak, M. and Korkusuz, F., Evaluation of body composition using three different methods compared to dual-energy X-ray absorptiometry. Eur J Sport Sci, 9(3), 181-190 (2009).

[18] Yaxley, A., Miller, M., Masters, S., Ahern, M. and Crotty, M., Body composition in older orthopaedic rehabilitation inpatients: Are field methods valid? Nutrition \& Dietetics, 67(3), 160165 (2010).

[19] Bottaro, M.F., Heyward, V.H., Bezerra, R.F. and Wagner, D.R., Skinfold method vs dual-energy $\mathrm{x}$-ray absorptiometry to assess body composition in normal and obese women. J Exerc Physiol Online, 5(2), 11-18 (2002).

[20] Durnin, J.V.G.A. and Womersley, J. Body fat assessment from body density and its estimation from skinfold thickness: Measurements on 481 men and women aged from 16 to 72 years. Br J Nutrition, 32, 77-99 (1974).

[21] Wagner, D.R. and Heyward, V.H., Techniques of body composition assessment: a review of laboratory and field methods. Res Q Exerc Sport, 70, 135-149 (1999).

[22] Dezenberg, C.V., Nagy, T.R., Gower, B.A., Johnson, R. and Goran, M.I., Predicting body composition from anthropometry in pre-adolescent children. International journal of obesity, 23(3), 253-259 (1999).

[23] Cameron, N., Griffiths, P.L., Wright, M.M., Blencowe, C., Davis, N.C., Pettifor, J.M. and Norris, S.A., Regression equations to estimate percentage body fat in African prepubertal children aged $9 \mathrm{y}$. The American journal of clinical nutrition, 80(1), 70-75 (2004).

[24] Siri, W.E., Body volume measurement by gas dilution. In J. Brozek \& A. Henschel (Eds.), Techniques for measuring body composition. Washington, DC: National Academy of Sciences, National Research Council, 1961, pp. 108-117. 
[25] Heyward, V.H. and Stolarczyk, L.M., Applied Body Composition Assessment. Champaign, IL; Human Kinetics, USA, 1996.

[26] International Standards for Anthropometric Assessment (ISAK). International Standards for Anthropometric Assessment. ISAK, Canberra, 2001, pp. 21-66.

[27] Siri, W.E., The gross composition of the body. "Advances in Biological and Medical physics" (Ed. C.A., Tobias, J.H., Lawrence), New York: Academic Press, 1956, 4, pp. 239-280.

[28] American College of Sports Medicine. ACSM's guidelines for exercise testing and prescription.6th ed. Media, PA: Williams \& Wilkins, 2000, pp. 214.

[29] Martin, A.D., Ross, W.D., Drinkwater, D.T., and Clarys, J.P., Prediction of body fat by skinfold caliper: assumptions and cadaver evidence. International journal of obesity, 9, 31-39 (1984). 